\title{
Crashproofing the Original NoSQL Key-Value Store
}

\section{TERENCE KELLY}

BEFORE TERMINALS, WE ALL USED PUNCH CARDS, WHICH WERE ORIGINALLY DEVELOPED TO CONTROL LOOMS. EARLY MAINFRAMES WOULD' PRODUCE A SLEATER EACH TIME YOU RAN YOUR CODE. EVENTUALLY WE GOT THEM TO STOP. WE HAD ENOUGH SWEATERS.

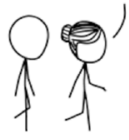

https:I/xkcd.com/2324I

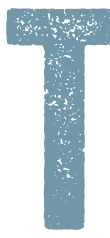

his episode of Drill Bits unveils a new crashtolerance mechanism that vaults the venerable gdbm database into the league of transactional NoSQL data stores. We'll motivate this upgrade by tracing gdbm's history. We'll survey the subtle science of crashproofing, navigating a minefield of traps for the unwary. We'll arrive at a compact and rugged design that leverages modern file-system features, and we'll tour the production-ready implementation of this design and its ergonomic interface.

\section{ENDURING PATTERNS FOR DURABLE DATA}

Ken Thompson wrote the original dbm amid a storied golden age. Sparked by Unix, software creativity flourished at Bell Labs in the 1970s, producing an ecosystem ${ }^{10}$ that remains vibrant decades later. The parent company's ambient technology may have inspired staff to re-imagine data organization, with profound consequences:

IBM OS builders worked in a place where data lived on unit record equipment (80-column cards, 132-column printers, etc.] and built a file system that looked like that. Ken and Dennis worked in a phone company where data traveled in streams over wires, and built a system in which files looked like that. $^{3}$ 
Unstructured byte streams from files and pipes forced applications to impose structure. Many opted for the easygoing key-value paradigm-often a good choice if a relational database would be overkill and fixed layout would be too rigid. Enduring key-value patterns from the early years include self-describing text formats ${ }^{2}$ and the built-in associative arrays of AWK and its descendants.

Thompson's dbm library provided a persistent key-value abstraction. ${ }^{16}$ Client programs manipulated pairs of key and value strings via a store()/fetch() interface; under the hood dbm managed an extensible hash table embedded in a file.

Philip Nelson implemented GNU gdbm in 1990. Since then maintainers Justin Downs and Sergey Poznyakoff have updated gdbm to exploit new Unix features such as writev() and mmap(). Release 1.20 (July 2021) improves performance via a complete rewrite of the hash-table bucket cache. Users and client software maintainers often help with testing.

Thanks to its maturity and ease of use, gdbm has become an indispensable workhorse. On some Linux distributions, one in six software packages depends directly or indirectly on libgdbm; prominent examples include man- $d b$ the manual pages], several Apache modules, and popular interfaces for Python and Perl. A survey of nearly 200,000 Debian systems found that 60 percent regularly use libgdbm. ${ }^{4}$

Unfortunately, crash vulnerability, the Achilles' heel of the $\mathrm{dbm}$ family, has been driving some potential users to alternative data stores ever since the 1990s. An untimely power failure, operating-system kernel panic, or process 
crash could maim a gdbm database. Applications that must tolerate crashes have therefore used "transactional" NoSQL data stores such as Berkeley DB" ${ }^{11}$ or LevelDB, or full-blown relational databases supporting ACID transactions. Now, however, you can have your gdbm and crash it too.

\section{SUCCEEDING AGAINST FAILURES}

Tolerating a crash means restoring persistent data to a consistent state that satisfies all relevant correctness criteria in all software layers, including the application. Consistency means more than merely the absence of corruption in lower layers: Consider an imprudently implemented banking application that intends to decrement one account balance and increment another by the same amount. A crash occurs between these two steps, and by chance it has the same effect as an orderly (though ill-timed) shutdown. Data integrity is impeccable at the storage, file-system, and database layers, but the application-level "conservation-of-money" invariant is violated.

Applications prevent crash-induced inconsistency by using transactions that perform multiple changes atomically, even in the presence of failures. An application must ensure that both the before and after states are consistent; a transaction mechanism guarantees that one or the other can be recovered following a crash. Unfortunately, reliable implementations are elusive: Mature, widely used transactional data stores don't always tolerate crashes. ${ }^{17}$

Little wonder. Portably implementing failure-atomic 
part from technical difficulties, conflicting priorities also undermine reliability updates atop Unix-like operating systems is remarkably difficult:12 File systems provide vague and often weak post-crash guarantees, which furthermore depend on configuration details. The Posix system calls that modify files and synchronously persist the changes to durable media-write()Ifsync() and mmap()Imsync()-are not atomic. For example, an attempted two-byte write() may return 1 , meaning that the first byte may become persistent at any time but we must try again to write() the second byte; meanwhile, of course, a crash may occur. The list of pitfalls goes on: Without explicit ordering constraints from fsync() or msync(), the operating system may push file modifications from main-memory buffers down to durable storage at any time and in any order. A crash may leave files corrupted but not obviously so. That's just the tip of the iceberg; Pillai et al. describe further challenges. ${ }^{13}$

Apart from technical difficulties, conflicting priorities also undermine reliability. Many data stores offer rich functionality, which requires complex code, which harbors bugs. Worse yet, many databases strive for performance to the detriment of crash tolerance-the main motivation for transactions. With databases as with parachutes, excessive speed compromises safety.

\section{CRASH-TOLERANCE PROTOCOL}

Crash tolerance in gdbm begins with a naïve protocol that is obviously slow and subtly flawed. Fixing the flaws shows that crashproofing is tricky but need not be esoteric. Fixing the performance problem leverages an existing solution to an unrelated problem. 
TABLE 1: DOCUMENTATION FOR MODERN SYSTEM CALLS AND UTILITIES

URLs append suffixes below to https://man7.org/linux/man-pages/

\begin{tabular}{ccc} 
man2/close.2.html & man3/malloc.3.html & man3/raise.3.html \\
man3lfree.3.html & man2/mmap.2.html & man3/realpath.3.html \\
\hdashline man2/fsync.2.html & man2/msync.2.html & man2/write.2.html \\
\hdashline man2lioctl_ficlone.2.html man2lopen.2.html & man2/writev.2.html \\
\hdashline man1/cp.1.html & man1/dd.1.html & man8/filefrag.8.html \\
\hline
\end{tabular}

A gdbm database resides in a data file. Traditionally, applications call gdbm_sync() to persist a database from volatile memory down through the file system onto durable storage media. This slow operation makes sense only when the database is consistent. Therefore, the existing gdbm_sync() function serves an additional purpose when crash tolerance is enabled: It tells gdbm that the database is consistent and suitable for crash recovery.

Our first (incorrect) attempt at crash tolerance is simply to copy the data file immediately after gdbm_sync(), and then fsync() the copy; post-crash recovery uses the most recent copy. This approach is broken in two subtle ways: First, copies might be lost in a crash because their directory entries were not persisted. Second, after a crash it's uncertain whether the most recent copy-and-fsync() completed successfully; the copy might be malformed.

To solve the first problem, we ensure that a copy is visible in the file system after a crash by calling fsync() on the copy itself and on every directory on the realpath() between the copy and the root. To solve the second problem, we mark copies containing consistent data using their permission bits. To make a copy we: open() it write-only; fill it with the contents of the gdbm data file; fsync() it; then 
change its permission bits to read-only; and finally fsync() the copy again to persist its permission bits. Crucially, file systems ensure that the metadata operation in the last step is failure-atomic and that the two fsync() calls persist data in program order. Post-crash recovery uses the most recent readable copy, which is sure to be consistent and persistent because it became readable after it was successfully filled with a consistent database and fsync()'d.

While correct, the protocol sketched above is impractical because it physically copies the entire data file on every gdbm_sync(). Copying a large data file to commit a few minor changes would be wasteful. The performance cost should be bounded not by the size of the entire database ["O[data]"] but by the size of changes between consecutive gdbm_sync() calls ["O(delta]"].

Fortunately, modern file systems support an efficient copy operation: Reflink cloning was motivated by virtual machines, which employ virtual disks that reside in files in a hypervisor's file system. Cloning reduces the overhead of multiple files containing near-identical copies of a virtual disk. Cloning doesn't physically copy storage blocks when a logical copy is made. Instead, the new clone shares data blocks with the original file. A copy-on-write mechanism ensures that subsequent changes to either file do not affect the other. The overall cost in time and storage grows with the changes to both files after the clone is made: $O$ [delta], not $O$ (data].

Figure 1 illustrates creating then changing a clone. On a Linux system with $4 \mathrm{KiB}$ file-system blocks, I filled a 100 -block file with random bytes, then cloned it with the commands 


\section{FIGURE 1: EXTENT MAPS OF ORIGINAL FILE AND CLONE}

(a) immediately after clone created, before changes to clone

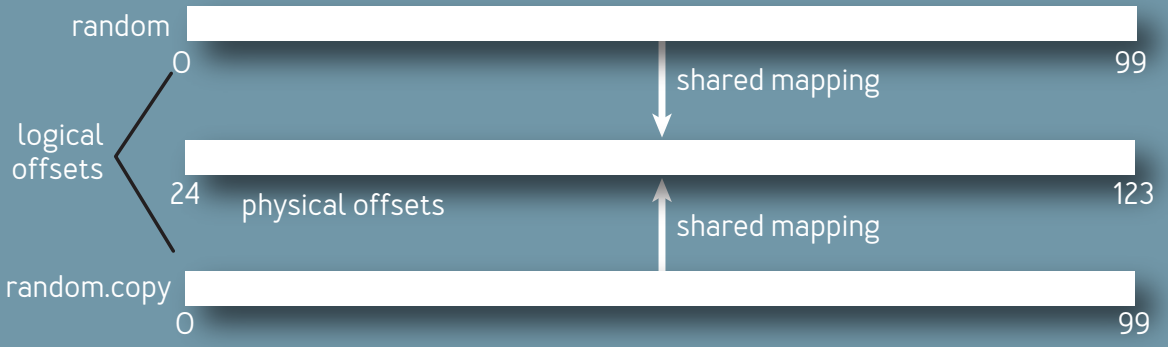

(b) after write[]s to blocks 7 and 47 of clone

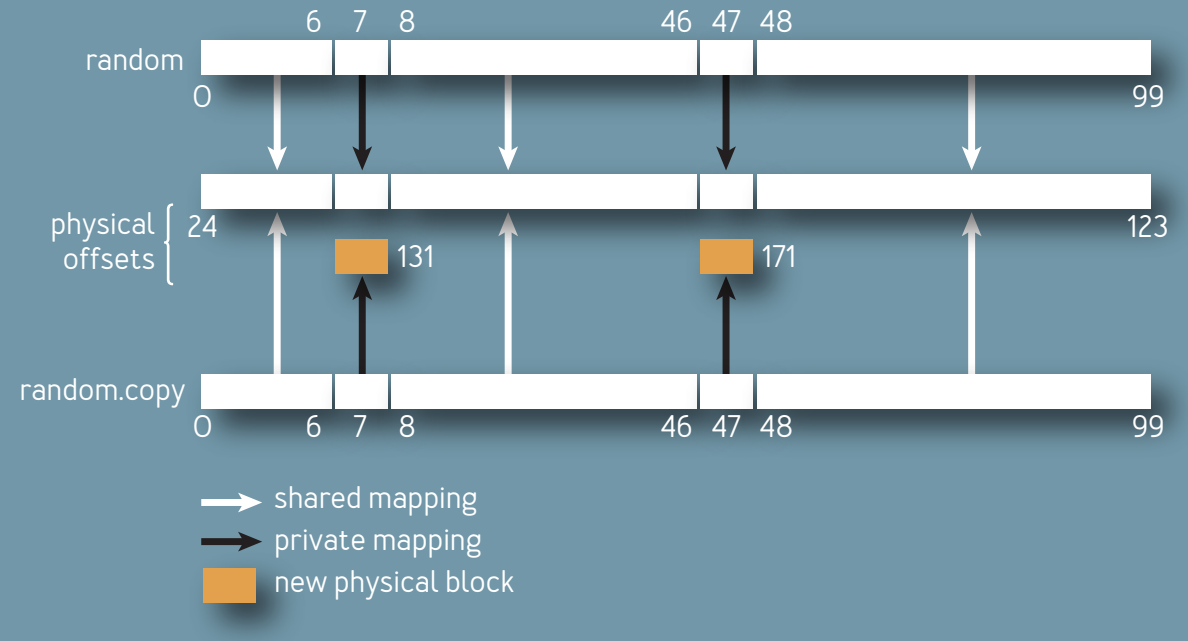

dd if=/dev/urandom bs=4096 count=100 > random $\mathrm{cp}$--reflink=always random random.copy

Figure 1a shows how the files map logical to physical blocks, courtesy the filefrag utility. Each file contains a 
single 100-block extent (range of blocks), and both files map the same logical extent to the same physical extent (i.e., they share storage]. Next I modified logical blocks 7 and 47 of the clone, for which copy-on-write allocated two new physical blocks (figure $1 \mathrm{~b}$ ). Both files now have five extents: three large shared extents and two private single-block extents; 98 percent of each file is backed by shared physical blocks.

Cloning is currently available on three file systems: OCFS2 [Oracle Cluster File System version 2), Btrfs, and XFS. For convenience we may install one of these reflink-capable file systems inside an ordinary file on a different file system such as ext4; the gdbm crash-tolerance documentation explains how..$^{15}$ Critical operations such as cloning and fsync() will work as reliably as if the reflink-capable file system had been installed directly on dedicated storage. ${ }^{6}$

Crashproofing via cloning offers different performance tradeoffs than transactions based on write-ahead logging. Reflink copy-on-write tracks changes to blocks, which are typically $4 \mathrm{KiB}$, whereas many transaction systems track changes at finer granularity. Workload affects performance differently in these approaches.

\section{IMPLEMENTATION DETAILS}

To prototype crash-tolerant gdbm I changed four source files in release 1.20, adding fewer than 200 lines of code in total. I tested my prototype against injected crashes; an earlier implementation of the same basic protocol survived tens of thousands of power failures. ${ }^{9}$ Original gdbm author Phil Nelson and maintainer Sergey Poznyakoff reviewed my protocol design. Poznyakoff reviewed my code, repeated my 


\section{key-value store}

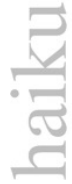

crashproof KVS? add ioctl(FICLONE)

to $\mathrm{gdbm}$ tests, and merged my prototype into release 1.21.

Applications "opt in" to crash tolerance by passing gdbm_failure_atomic() a pointer to a gdbm database and the names of two snapshot files that will hold clones of the main data file; all three files must reside on the same reflink-capable file system. The gdbm_failure_atomic() call creates two empty write-only snapshot files with the given names and fsync()s them and their enclosing directories up to the root. After gdbm_failure_atomic() returns, subsequent calls to gdbm_sync() use ioctI(FICLONE) to deposit clones of the main database file in one or the other snapshot file according to the protocol described earlier, alternating between the two.

Following a crash, applications recover by calling gdbm_ latest_snapshot() to identify the most recently modified snapshot file. This file, which contains a consistent database reflecting the most recent successful gdbm_ sync() call, simply replaces the main gdbm data file.

Because gdbm doesn't use write-ahead logs, there's no need to "garbage collect" logs that pile up during failurefree operation and no need to replay logs to reconstruct the database during post-crash recovery.

Crash tolerance depends on the proper functioning of system calls and of file and storage systems; all bets are off if, say, a storage device misbehaves after a power failure. ${ }^{18}$ Furthermore the gdbm library can't protect an application from corrupting its own database-a substantial risk for $\mathrm{ClC}++$ programs but a small risk for the many Python and Perl programs that use gdbm.

It's easy to understand crash-tolerant gdbm and use it 
correctly because it simply strengthens the semantics of a familiar interface: gdbm_sync() has always meant "persist the database"; crash tolerance adds, "and failureatomically clone it too." All changes to a gdbm database between consecutive gdbm_sync() calls constitute a transaction. By contrast, a begin()- and end()transaction interface is more error prone: Bookends such as malloc()/free(), lock()/unlock(), and open()/ close() are easy to mismatch.

\section{DRILLING DEEPER}

Like packing your own parachute, rolling your own crash tolerance is foolhardy without proper training and supervision. An off-the-shelf transaction system is usually better-if it's fit for purpose. ${ }^{17}$ To be a smart shopper and a smart user, study the design and implementation of failureatomic update mechanisms. Learn how to audit and break them. Understand how they depend on operating systems, file systems, and storage. 12,13,18 If you rely on transactions, train as you fight: Test your entire hardware-software stack against the failures that it must survive in production. ${ }^{9}$

\section{Bits}

Grab gdbm release 1.21 from https:/lftp.gnu.orglgnul gdbm/gdbm-1.21.tar.gz. In file gdbmsync.c, check out the implementations of user-visible function gdbm_failure atomic() and internal function_gdbm_snapshot(), called after gdbm_sync() finishes its traditional job. If you need a crash-tolerant key-value store, consider the new gdbm after reading all relevant documentation.14,15 


\section{Drills}

1. Standardized performance tests for databases abound; examples include the TPC benchmarks. Try to find crashtolerance tests.

2. Find an application that already uses gdbm. Modify it to exploit crash tolerance.

3. Find software that uses some other transactional keyvalue store and modify it to use crash-tolerant gdbm. Test both against realistic failures. ${ }^{9}$ If both pass, compare performance. Construct workloads for which each is faster than the other. Measure sustainable long-term steady states; don't allow write-ahead logs to pile up.

4. If a distant customer asks a bank to transfer money from a savings account to a checking account, what's the relationship between the balance transfer transaction and the bank's reply informing the customer that the money has been moved? What can go wrong if the reply and the transaction aren't properly coordinated?

5. Reliability and security are closely related. What principles from the economics of computer security ${ }^{1}$ apply to reliability? For example, who should evaluate crash-tolerance claims: database vendors, independent researchers, or end users?

6. What are the pros and cons of using raise(SIGKILL) to "abort a transaction" in gdbm?

7. One way to build a persistent hash table is to begin with an in-memory hash table [for example, a C++ STL <map >], and make it persistent. ${ }^{8}$ Adding crash tolerance as described earlier isn't difficult. ${ }^{7}$ Compare that approach to transactional key-value stores such as gdbm. Which supports a more natural programming style? 


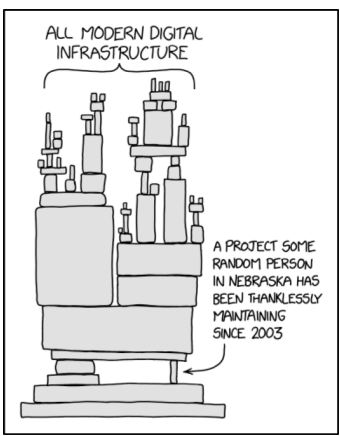

https:IIxkcd.com/2347I

\section{Acknowledgments}

GDBM author Phil Nelson, GDBM maintainer Sergey

Poznyakoff, and Programming Pearls author Jon Bentley provided valuable feedback on drafts of this column. Lionel Debroux answered questions about key-value stores. XFS developer Christoph Hellwig answered questions about reflink cloning.

\section{References}

1. Anderson, R. 2001. Why information security is hard-an economic perspective. In Annual Computer Security Applications Conference [ACSAC], December; https:II www.acsac.org/2001/papers/110.pdf.

2. Bentley, J. 1987. Programming pearls: self-describing data. Communications of the ACM 30(6), 479-483; https:I/doi.org/10.1145/214762.315733. (Chapter 4 in More Programming Pearls, Addison-Wesley, 1988).

3. Bentley, J. August 2019. E-mail to colleague. Ken Thompson and Dennis Ritchie wrote the first UNIX operating system.

4. Debian popularity contest. July 2021; https:I/popcon. debian.orgl.

5. Ghemawat, S., Dean, J. 2021. LevelDB; https:Igithub.coml googlelleveldb.

6. Hellwig, C. July 2021. Personal communication. Requirements for block devices and file-system semantics guarantee that filesystem-in-file installations function properly.

7. Kelly, T. 2019. Good old-fashioned persistent memory. Usenix ;login: 44(4), 29-34; https:IIwww. usenix.orgl system/files/loginlarticles/login_winter19_08_kelly.pdf. 
8. Kelly, T. 2019. Persistent memory programming on conventional hardware. acmqueue 17(4); https:/lqueue.acm.org/detail.cfm?id=3358957.

9. Kelly, T. 2020. Is persistent memory persistent? Communications of the ACM, 63[9], 48-54; https:I/dl.acm. org/doi/10.1145/3397882. To avoid paywall: acmqueue 18(2); https://queue.acm.org/detail.cfm?id=3400902.

10. Kernighan, B. W., Pike, R. 1984. The UNIX Programming Environment. Prentice Hall.

11. Oracle Berkeley DB. July 2021; https:I/www.oracle.com/ databaselberkeley-dbl.

12. Pillai, T. S., et al. 2014. All file systems are not created equal: on the complexity of crafting crash-consistent applications. In Proceedings of the 11 th Usenix Symposium on Operating Systems Design and Implementation (OSDI); https://www.usenix.org/system/files/conferencel osdi14losdi14-paper-pillai.pdf.

13. Pillai, T. S., et al. 2015. Crash consistency. acmqueue 13(7); https:I/dl.acm.org/doilpdf/10.1145/2800695.2801719.

14. Poznyakoff, S. 2021. Crash-tolerant gdbm (September]. Release 1.21 (September 2021). Source tarball: https://ftp.gnu.org/gnu/gdbm/gdbm-1.21.tar.gz. Diff against 1.20: https:/lgit.gnu.org.ualgdbm.git/ rawdiff $/$ ?id=v1.21Eid2=v1.20

15. Poznyakoff, S. 2021. Crash tolerance. gdbm Manual [July]; https://www.gnu.org.ua/softwarelgdbm/current/ Crash-Tolerance.html.

16. Unix dbm manual; http://man.cat-v.org/unix_7th/3/dbm.

17. Zheng, M., Tucek, J., Huang, D., Qin, F., Lillibridge, M., Yang, E. S., Zhao, B. W., Singh, S. 2014. Torturing databases for fun and profit. In Proceedings of the $11^{\text {th }}$ 
Usenix Symposium on Operating Systems Design and Implementation [OSDI]; https://www.usenix.org/system/ files/conferencelosdi14losdi14-paper-zheng_mai.pdf. (Note that an errata sheet is provided separately.) 18. Zheng, M., Tucek, J., Qin, F., Lillibridge, M. 2013. Understanding the robustness of SSDs under power fault. In Proceedings of the $11^{\text {th }}$ Usenix Conference on File and Storage Technologies (FAST); https:Ilwww. usenix. orglsystem/files/conferencelfast13/fast13-final80.pdf.

At the drop zone, former skydiver Terence Kelly [tpkelly@acm.org]went by the nickname "Crash."

Copyright $(2021$ held by ownerlauthor. Publication rights licensed to ACM.

\section{SHAPE THE FUTURE OF COMPUTING!}

Join ACM today at acm.org/join

\section{BE CREATIVE. STAY CONNECTED. KEEP INVENTING.}

Association for

Computing Machinery

Advancing Computing as a Science \& Profession 